\title{
THE INTEGRAL REPRESENTATION OF UNBOUNDED SELF-ADJOINT TRANSFORMATIONS IN HILBERT SPACE*
}

\author{
BY \\ FREDERICK RIESZ AND E. R. LORCH
}

INTRODUCTION

In this note we are concerned with unbounded self-adjoint transformations in Hilbert space. Denoting such a transformation by $A$ we give two short demonstrations of the facts associated with the formula

$$
A=\int_{-\infty}^{\infty} \lambda d E_{\lambda}(A) \text {. }
$$

That is, we establish the integral representation of $A$ by means of the resolution of the identity $E_{\lambda}(A)$ corresponding to $A$ (for definitions, see below). The facts in question for the case of bounded transformations have been known in substance since the appearance in 1906 of Hilbert's memoir on integral equations. The unbounded case on the other hand has received attention only in recent years. $\dagger$ Each of the three methods given to establish (1) for unbounded transformations is based to a certain extent on the theory of bounded transformations, indeed to the extent of making use of the transformation $(A-i E)^{-1}$; but none of these methods exploits formula (1) for the bounded case and its immediate consequences systematically. Our purpose is to show that by doing so the proofs can be shortened considerably.

The fundamental idea of the first proof is to establish the existence of an orthogonal system of closed linear manifolds sweeping out the whole space and such that $A$ behaves like a bounded transformation in each manifold considered as a Hilbert space. Thus for each manifold, formula (1) is valid; the behavior of $A$ in the original space is obtained from its behavior in each manifold by a simple synthesis.

The motivating idea of the second proof is to introduce a bounded selfadjoint transformation $D$ whose resolution of the identity is topologically

\footnotetext{
* Presented to the Society, September 10, 1935; received by the editors September 26, 1935.

$\dagger$ Cf. J. von Neumann, Allgemeine Eigenwerttheorie Hermitescher Funktionaloperatoren, Mathematische Annalen, vol. 102 (1929), pp. 49-131; M. H. Stone, Linear transformations in Hilbert space, Proceedings of the National Academy of Sciences, vol. 15 (1929), pp. 198-200 and 423-425; F. Riesz, Über die linearen Transformationen des komplexen Hilbertschen Raumes, Acta Litterarum ac Scientiarum (Szeged), vol. 5 (1930), pp. 23-54.
} 
equivalent to that of $A$, or, in the language of the operational calculus, $D$ is a monotone function of $A$. The particular function which we use is $\lambda|\lambda| /\left(1+\lambda^{2}\right)$. On first thought one is tempted to use the function $\arctan \lambda$, but the former function, being practically rational, leads to easier calculations than the latter.

The paper is divided into five parts. In $\$ 1$ we repeat the definition of a self-adjoint transformation and establish directly from this definition a lemma of interest in the unbounded case. $\$ 2$ is devoted to bounded transformations. We state precisely what facts, known for over twenty years, we use in the subsequent proofs. In $\$ \S 3$ and 4 we carry out the first proof. $\$ 5$ is devoted to the second.

1. The definition of a self-adjoint transformation; a lemma. We use the symbols $A, B, C$, etc., to represent linear transformations defined on a subset of Hilbert space $\mathfrak{S}$ which is dense in $\mathfrak{S}$ (the subset may be the whole space); the symbols $\Delta_{A}, \Delta_{B}, \Delta_{C}$, etc., represent the domains of definition of these transformations; and $f, g, h$ represent elements of $\mathfrak{S}$. In later sections, functions of the real variable $\lambda$ will be denoted by $R(\lambda), S(\lambda), T(\lambda)$, etc. We find with respect to a transformation $A$ all pairs of elements $\left[f, f^{*}\right]$ satisfying the equation

$$
(A g, f)=\left(g, f^{*}\right) \text { for all } g \in \Delta_{\Lambda} \text {. }
$$

The transformation $A^{*}$ defined by the equation $A^{*} f=f^{*}$ is called the adjoint of $A$. It is easy to verify in (2) that $A^{*}$ is a linear transformation. In addition, $A^{*}$ has the property of closure or is a closed transformation; that is, if $f_{n} \in \Delta_{A^{*}}(n=1,2, \cdots)$, and if $f_{n} \rightarrow f, A^{*} f_{n} \rightarrow f^{*}, \dagger$ then $f \in \Delta_{A^{*}}$ and $A^{*} f=f^{*}$. This results from the continuity of the inner product $(h, k)$. If $\Delta_{A}=\Delta_{A^{*}}$ and if $A=A^{*}$ in this common domain, we say that $A$ is self-adjoint.

We shall derive from the foregoing a lemma which is of use in the subsequent discussion. It is convenient first to introduce a few definitions. Let $\mathfrak{M} \neq \mathfrak{S}$ be a closed linear manifold, and let $A$ be a transformation defined for every element of $\mathfrak{M}$ and transforming $\mathfrak{M}$ into a subset of itself. For our present purposes, $A$ may or may not be defined outside of $\mathfrak{M}$. An expression of the type " $A$ is self-adjoint in $\mathfrak{M}$ " will mean that the transformation $A$ considered in the Hilbert space (or space of a finite number of dimensions) $\mathfrak{M}$ is self-adjoint. The behavior of $A$ outside of $\mathfrak{M}$ does not in any way affect its characterization in $\mathfrak{M}$.

Let $\mathfrak{M}_{1}, \mathfrak{M}_{2}, \cdots$ be an orthogonal sequence of closed linear manifolds, that is, such that if $f_{i} \in \mathfrak{M}_{i}$, then $\left(f_{i}, f_{j}\right)=0, i \neq j$. We use the symbol $\sum_{\alpha=1}^{\infty} \mathfrak{M}_{\alpha}$ to denote the smallest closed linear manifold containing each $\mathfrak{M}_{i}$. Evidently

$\dagger$ The symbol $f_{n} \rightarrow f$ indicates that $\lim _{n \rightarrow \infty}\left\|f-f_{n}\right\|=0$. 
$\sum_{\alpha=1}^{\infty} \mathfrak{M}_{\alpha}$ contains precisely all the elements of the form $f_{1}+\cdots+f_{n}$ and their limits. We have the

Lemma. Let $\mathfrak{M}_{1}, \mathfrak{M}_{2}, \cdots$ be an orthogonal sequence of closed linear manifolds such that $\sum_{\alpha=1}^{\infty} \mathfrak{M}_{\alpha}=\mathfrak{G}$. If $f \in \mathfrak{S}$, let $f_{i}$ denote the projection of $f$ on $\mathfrak{M}_{i}$. Let $A_{i}$ be transformations which are self-adjoint in $\mathfrak{M}_{i}$. Then there exists precisely one self-adjoint transformation $A$ which is identical with $A_{i}$ in $\mathfrak{M}_{i}$. The domain of $A, \Delta_{A}$, consists of all $f \in \mathfrak{S}$ such that $\sum_{\alpha=1}^{\infty}\left\|A_{\alpha} f_{\alpha}\right\|^{2}$ converges; if $f_{\epsilon} \Delta_{A}$, then $A f=\sum_{\alpha=1}^{\infty} A_{\alpha} f_{\alpha}$.

We show first that the transformation $A$ as defined above is self-adjoint. If $f \in \Delta_{A}$, then clearly $A\left(f-f_{i}\right)$ is orthogonal to any element in $\mathfrak{M}_{i}$. This means that $A^{*}$ is defined in $\mathfrak{M}_{i}$ and equal to $A$ in $\mathfrak{M}_{i}$, since we have for any $f \in \Delta_{A}$ and $g \in \mathfrak{M}_{i}$

$$
(A f, g)=\left(A\left(f-f_{i}\right), g\right)+\left(A f_{i}, g\right)=\left(f_{i}, A g\right)=(f, A g) .
$$

Since $A^{*}$ is a closed transformation, $\Delta_{A^{*}} \supseteq \Delta_{A}$ and $A^{*}=A$ in $\Delta_{A}$. Now let $h \in \Delta_{A^{*}} ;$ then

$$
\left(A^{*}\left(h-h_{i}\right), A h_{i}\right)=\left(\left(h-h_{i}\right), A^{2} h_{i}\right)=0
$$

and hence

$$
\left\|A^{*} h\right\|^{2}=\left\|A^{*}\left(h-h_{i}\right)\right\|^{2}+\left\|A h_{i}\right\|^{2} .
$$

In turn

$$
\left\|A^{*} h\right\|^{2} \geqq \sum_{\alpha=1}^{\infty}\left\|A h_{\alpha}\right\|^{2}
$$

Thus $h \in \Delta_{A}$, and since $A^{*}$ is a closed transformation, $A h=A^{*} h$.

To complete the proof of the lemma, we show that there exists only one self-adjoint transformation which is equal to $A_{i}$ in $\mathfrak{M}_{i}$. Let $B$ be any transformation having the requisite properties. Since $B$ is a closed transformation, $\Delta_{B} \supseteq \Delta_{A}$ and $A=B$ in $\Delta_{A}$. If $h \in \Delta_{B}$, we repeat the argument in (3) and what immediately follows, replacing $A^{*}$ by $B$, and show that $h \in \Delta_{A}$. Hence $A$ and $B$ are identical.

2. The operational calculus for the bounded case. Below appears a résumé of known facts concerning bounded self-adjoint transformations which form the basis of the subsequent discussion; no proofs are given.

A projection $P$ is a self-adjoint transformation such that $\Delta_{P}=\mathfrak{S}, P^{2}=P$. A family of projections, $E_{\lambda},-\infty<\lambda<\infty$, is said to be a resolution of the identity if it possesses the following properties: (1) $E_{\lambda} E_{\mu}=E_{\mu} E_{\lambda}=E_{\lambda}, \lambda \leqq \mu$; 
(2) $E_{\lambda} \rightarrow 0$ (the zero transformation) or $E$ (the identity transformation) as $\lambda \rightarrow-\infty$ or $\infty$; and (3) $E_{\lambda+0}=E_{\lambda}$.

A transformation $A$ is said to be bounded if there exists a constant $c$ such that $\|A f\| \leqq c\|f\|, f \in \Delta_{A}$. The least constant of this type is said to be the bound of $A$; it is denoted by $M_{A}$. If $A$ is bounded and possesses the property of closure, it is easy to see that $\Delta_{A}=\mathfrak{S}$. If $A$ is bounded and self-adjoint, it has the following properties:

(a) Corresponding to $A$, there exists a unique resolution of the identity $E_{\lambda}(A)$ enabling us to write equation (1); the integration here is of the Stieltjes type and is to be interpreted in an obvious manner. The boundedness of $A$ is equivalent to the boundedness of the quadratic form $(A f, f)$ under the condition that $\|f\|=1$. If $m$ and $M$ denote the greatest lower and the least upper bound of this form, then $E_{\mu}(A)=0, \mu<m$, and $E_{\mu}(A)=E, \mu \geqq M$. The bound of $A$ is the greater of the numbers $|m|$ and $|M|$. The integral (1) may always be replaced by an integral over a finite interval containing the points $\lambda=m$ and $\lambda=M$ in its interior. In case $E_{m}(A)=E_{m-0}(A)$, and this will be the case with which we have to deal, we may write

$$
A=\int_{m}^{M} \lambda d E_{\lambda}(A) .
$$

(b) If $R(\lambda)$ represents a real function continuous for $m \leqq \lambda \leqq M$, the integral $\int_{-\infty}^{\infty} R(\lambda) d E_{\lambda}(A)$ represents a bounded self-adjoint transformation denoted by $R(A)$. In particular if $R(\lambda)=1, R(A)=E$. We point out that the behavior of $R(\lambda)$ outside of the interval $m \leqq \lambda \leqq M$ is not of consequence in defining $R(A)$, since for such values of $\lambda, E_{\lambda}(A)$ is constant.

(c) $E_{\lambda}(A)$ and $R(A)$ are permutable with every bounded transformation permutable with $A$.

(d) For two continuous functions $R(\lambda)$ and $S(\lambda)$,

$$
\int_{-\infty}^{\infty} R(\lambda) d E_{\lambda}(A) \cdot \int_{-\infty}^{\infty} S(\lambda) d E_{\lambda}(A)=\int_{-\infty}^{\infty} R(\lambda) S(\lambda) d E_{\lambda}(A) .
$$

3. The first proof: the transformations $B$ and $C$. Let $A$ be an unbounded self-adjoint transformation, that is to say, there does not exist a constant $c$ such that $\|A f\| \leqq c\|f\|$, for all $f \in \Delta_{A}$. By an ingenious device due to J. von Neumann, $\dagger$ we shall introduce the transformations $B$ and $C$ which are the imaginary and real parts respectively of the transformation $(A-i E)^{-1}$. Con-

$\dagger$ Cf. J. von Neumann, Über adjungierte Funktionaloperatoren, Annals of Mathematics, vol. 33 (1932), pp. 294-310. Other methods for introducing the transformations $B$ and $C$ may be found in the papers referred to in the second foot-note on p. 331 . 
sider the set of all pairs $\{f, g\}$ of elements in $\mathfrak{S}$. These pairs form a Hilbert space $\mathfrak{S}^{\prime}$ if the fundamental operations are performed as follows:

and

$$
\begin{aligned}
\alpha\{f, g\} & =\{\alpha f, \alpha g\}, \quad \alpha \text { a complex number; } \\
\left\{f_{1}, g_{1}\right\}+\left\{f_{2}, g_{2}\right\} & =\left\{f_{1}+f_{2}, g_{1}+g_{2}\right\} ;
\end{aligned}
$$

$$
\left(\left\{f_{1}, g_{1}\right\},\left\{f_{2}, g_{2}\right\}\right)=\left(f_{1}, f_{2}\right)+\left(g_{1}, g_{2}\right) \text {. }
$$

Since $A$ is linear and closed, the manifold of all elements $\{A f, f\}, f \in \Delta_{A}$, in $\mathfrak{W}^{\prime}$ is linear and closed. The elements of its orthogonal complement are of the form $\{g,-A g\}$. In fact, let $\{g, h\}$ be orthogonal to each $\{A f, f\}$. Then

$$
(\{A f, f\},\{g, h\})=(A f, g)+(f, h)=0 .
$$

Since $A$ is self-adjoint, $g \in \Delta_{A}$ and $A g=-h$.

Let $h \epsilon \mathfrak{W}$; we express the element $\{h, 0\}$ in $\mathfrak{S}^{\prime}$ as the sum of its two orthogonal complements

This gives

$$
\{h, 0\}=\{A f, f\}+\{g,-A g\} .
$$

$$
A f+g=h, \quad f-A g=0 .
$$

Clearly $h$ determines $f$ and $g$ uniquely; in addition, the correspondences between $h$ and $g$ and between $h$ and $f$ are linear. Let $B$ and $C$ be the transformations defined by the equations $B h=g, C h=f$. The transformation $A^{2}$ is defined for all elements of the form $B h$; the transformation $A$ is defined for all elements of the form $C h$. The formulas (4) may now be written in the form

$$
A B=C
$$

and

$$
A C=E-B .
$$

If $h \in \Delta_{A}$, then one may apply the transformation $A$ to the formulas (4), which by the definition of $B$ and $C$ gives

$$
B A h=A g=A B h, \quad C A h=A f=A C h,
$$

that is,

$$
B A=A B \quad \text { and } \quad C A=A C .
$$

The two following equations may now be derived:

$$
C^{2}=C A B=A C B=(E-B) B=B-B^{2}
$$

and

$$
B C=B A B=A B B=C B .
$$


The transformations $B$ and $C$ are self-adjoint. Referring to (4), and with an evident notation, we have in turn for $B$ and $C$

$$
\begin{aligned}
\left(B h_{1}, h_{2}\right) & =\left(g_{1}, A^{2} g_{2}+g_{2}\right)=\left(A^{2} g_{1}+g_{1}, g_{2}\right)=\left(h_{1}, B h_{2}\right), \\
\left(C h_{1}, h_{2}\right) & =\left(f_{1}, A^{2} g_{2}+g_{2}\right)=\left(A f_{1}, A g_{2}\right)+\left(f_{1}, g_{2}\right)=\left(h_{1}-g_{1}, A g_{2}\right)+\left(A g_{1}, g_{2}\right) \\
& =\left(h_{1}, A g_{2}\right)=\left(h_{1}, C h_{2}\right) .
\end{aligned}
$$

The transformations $B$ and $C$ are bounded and $M_{B} \leqq 1, M_{C} \leqq 1$. This arises from the orthogonal decomposition of $\{h, 0\}$ into $\{A f, f\}$ and $\{g,-A g\}$, which gives

and so

$$
\|h\|^{2}=\|A f\|^{2}+\|f\|^{2}+\|g\|^{2}+\|A g\|^{2},
$$

$$
\|B h\|=\|g\| \leqq\|h\|, \quad\|C h\|=\|f\| \leqq\|h\| .
$$

Furthermore, by (7), the transformation $B=B^{2}+C^{2}$ is positive definite; more precisely,

$$
0 \leqq(B h, B h)+(C h, C h)=(B h, h) \leqq(h, h),
$$

and the case $(B h, h)=0$ can arise only if $g=B h=0, f=C h=0$, hence $h=A f+g=0$.

4. Conclusion of the first proof. Since $B h=0$ implies $h=0$ (see (9)), $E_{\lambda}(B)$, the resolution of the identity of $B$, satisfies the equation $E_{0}(B)=0$. This is an immediate consequence of the interpretation of the integral (1) where $A$ is to be replaced by $B$. Let $\mathfrak{M}_{n}(n=1,2, \cdots)$ be the closed linear manifold of all elements of the form $\left[E_{1 / n}(B)-E_{1 /(n+1)}(B)\right] h, h \in \mathfrak{W}$. Then by the first property of a resolution of the identity (see $\$ 2$ ), the sequence $\mathfrak{M}_{1}, \mathfrak{M}_{2}, \ldots$ is an orthogonal sequence. Referring to the first statement of this paragraph and to the fact that $E_{1}(B)=E$, we see that $\sum_{\alpha=1}^{\infty} \mathfrak{M}_{\alpha}=\mathfrak{S}$. We now proceed to show that the transformation $A$ is defined throughout $\mathfrak{M}_{n}$ and transforms this manifold into a subset of itself.

Let $F$ be any transformation permutable with $E_{\lambda}(B)$ for all $\lambda$. Then

$$
F\left[E_{1 / n}(B)-E_{1 /(n+1)}(B)\right]=\left[E_{1 / n}(B)-E_{1 /(n+1)}(B)\right] F,
$$

and consequently $F$ transforms $\mathfrak{M}_{n}$ into a subset of itself. Specifically $C$ and any function of $B$ transform this manifold into a subset of itself (see (8) and $\$ 2(\mathrm{c}))$. Keeping the value of $n$ fixed, let $R(\lambda)$ be the continuous function equal to $1 / \lambda$ for $1 /(n+1) \leqq \lambda \leqq 1 / n$ and constant otherwise. Then

$$
R(B) B=B R(B)=E
$$

in $\mathfrak{M}_{n}$ by $\S 2(\mathrm{~b})$ in conjunction with the equations

$$
E_{1 / n}(B) g=g, \quad E_{1 /(n+1)}(B) g=0,
$$


for any $g$ in $\mathfrak{M}_{n}$. In other words $R(B)$ and $B$ transform $\mathfrak{M}_{n}$ into itself in a one-to-one fashion and are inverses of each other in this manifold. Applying this to (5) we see that

$$
A=A B R(B)=C R(B) \text { in } \mathfrak{M}_{n} .
$$

Thus $A$ is defined throughout $\mathfrak{M}_{n}$. Furthermore, since $C$ and $R(B)$ transform $\mathfrak{M}_{n}$ into a subset of itself, $A$ does also.

In $\mathfrak{M}_{n}, A$ is a bounded self-adjoint transformation. For the sake of a simple notation, let us call the resolution of the identity of $A$ in this manifold $E_{\lambda, n}$. That is, $E_{\lambda, n}$ represents a family of projections in the Hilbert space (or space of a finite number of dimensions) $\mathfrak{M}_{n}$ having the familiar properties of a resolution of the identity. Let $E_{\lambda}$ represent the family of projections in the space $\mathfrak{E}$ which are identical with $E_{\lambda, n}$ in $\mathfrak{M}_{n}$ for every $n$. Then $E_{\lambda}$ is a resolution of the identity. The integral $\int_{-\infty}^{\infty} \lambda d E_{\lambda}$ represents a self-adjoint transformation. $\dagger$ Since for any $g \in \mathfrak{M}_{n}, E_{\lambda} g=E_{\lambda, n} g$, this transformation is identical with $A$ in $\mathfrak{M}_{n}$. Applying our lemma we obtain equation (1) as desired.

5. The second proof. $\S 3$ in its entirety is a prerequisite for this proof.

Consider $E_{\lambda}(C)$, the resolution of the identity of the transformation $C$ introduced above. Let $\mathfrak{M}_{-}$and $\mathfrak{M}_{+}$be the closed linear manifolds of all elements of the form $E_{0}(C) h$ and $\left[E-E_{0}(C)\right] h, h \in \mathfrak{W}$, respectively. Thus $\mathfrak{M}_{-}$is the orthogonal complement of $\mathfrak{M}_{+}$. Let $D$ be the unique transformation linear in $\mathfrak{g}$ which is defined by the equations

$$
D=-E+B=-A C \text { in } \mathfrak{M}_{-} ; \quad D=E-B=A C \text { in } \mathfrak{M}_{+} .
$$

Since $B$ and $C$ are permutable, $B$ and $E_{\lambda}(C)$ are permutable by $\S 2(\mathrm{c})$. Thus $B$, and hence also $D$, transform $\mathfrak{M}_{-}$into a subset of itself; a similar statement can be made for $\mathfrak{M}_{+}$. Since $D$ is self-adjoint when considered as a transformation in $\mathfrak{M}_{-}$or in $\mathfrak{M}_{+}, D$ is self-adjoint in the entire space $\mathfrak{W}$.

Equation (9) may be rewritten in the form

$$
0 \geqq-(h, h)+(B h, h)>-(h, h), \quad h \neq 0 .
$$

If we assume that $h \in \mathfrak{M}_{-}$, the central expression of this inequality is precisely $(D h, h)$. Similarly, for $h(\neq 0) \in \mathfrak{M}_{+}$,

$$
0 \leqq(D h, h)<(h, h) .
$$

Thus for $h \neq 0 \epsilon \mathfrak{W}$, writing $h=h_{1}+h_{2}, h_{1} \in \mathfrak{M}_{-}, h_{2} \in \mathfrak{M}_{+}$,

$\dagger$ We consider the manifolds $\left[E_{n}-E_{n-1}\right] h, h \in \mathfrak{S}, n=0, \pm 1, \pm 2, \cdots$. In each manifold the transformation defined by the integral $\int_{n-1}^{n} \lambda d E_{\lambda}$ is a bounded self-adjoint transformation. We define $\int_{-\infty}^{\infty} \lambda d E_{\lambda}$ to represent the unique self-adjoint transformation which in the manifold considered above is the transformation there given and whose existence is guaranteed by the lemma of $\$ 1$. It is clear that the system of integers could be replaced by any other infinite set of numbers possessing no finite limiting value but extending along the axis of reals indefinitely in both directions. 
$-(h, h)=-\left(h_{1}, h_{1}\right)-\left(h_{2}, h_{2}\right)<\left(D h_{1}, h_{1}\right)+\left(D h_{2}, h_{2}\right)=(D h, h)<(h, h)$.

It follows immediately that $D h=-h$ or $D h=h$ implies $h=0$. Thus by $\S 2$ (a)

$$
E_{-1}(D)=0, \quad E_{1-0}(D)=E,
$$

where $E_{\lambda}(D)$ obviously represents the resolution of the identity of $D$.

Let $\cdots<a_{-n}<\cdots<a_{0}=0<\cdots<a_{n} \cdots$ be a set of numbers converging on the left to -1 , on the right to 1 . Let $\mathfrak{N}_{i}$ be the manifold of all elements of the form $\left[E_{a_{i}}(D)-E_{a_{i-1}}(D)\right] h, h \in \mathfrak{W}$. The sequence $\mathfrak{N}_{0}, \mathfrak{N}_{1}, \mathfrak{N}_{-1}, \cdots$ is an orthogonal sequence and $\sum_{\alpha=-\infty}^{\infty} \mathfrak{R}_{\alpha}=\mathfrak{W}$.

Clearly $D$ and $B$ transform $\mathfrak{N}_{i}$ into a subset of itself. To prove that $C$ has the same property, it will be sufficient to show that $C$ and $D$ are permutable. If $h \in \mathfrak{M}_{+}$, we have

$$
C D h=C(E-B) h=(E-B) C h=D C h,
$$

since $C h \in \mathbb{M}_{+}$. A similar remark demonstrates that $C D h$ and $D C h$ are identical for $h \in \mathfrak{M}$. Since any element in $\mathfrak{S}$ may be expressed as the sum of two elements, the one in $\mathfrak{M}_{+}$, the other in $\mathfrak{M}_{-}, D$ and $C$ are permutable.

We now choose an $i \geqq 1$ and hold it fixed. If $h \in \mathfrak{N}_{i}$, then

$$
B h=\int_{-1}^{1}(1-\lambda) d E_{\lambda}(D) h .
$$

Allowing $R(\lambda)$ to be the continuous function equal to $(1-\lambda)^{-1}$ for $a_{i-1} \leqq \lambda \leqq a_{i}$ and constant otherwise, we may write

$$
R(D) B h=B R(D) h=h \text { for } h \in \Re_{i} .
$$

Hence

$$
A=A B R(D)=C R(D),
$$

which means that $A$ is defined throughout $\mathfrak{N}_{i}$ and transforms it into a subset of itself.

From (7), we derive the equation

$$
C^{2}=D-D^{2}
$$

valid in $\mathfrak{N}_{i}(i \geqq 1)$. We assert that since $(C h, h) \geqq 0, h \in \mathfrak{N}_{i}$,

$$
C=\int_{0}^{1}\left(\lambda-\lambda^{2}\right)^{1 / 2} d E_{\lambda}(D) \text { in } \Re_{i}
$$

More generally, we may state that if $G$ is a bounded self-adjoint transformation such that $(G h, h) \geqq 0, h \in \mathfrak{S}$, then there exists a unique bounded self-ad- 
joint transformation $F$ such that $F^{2}=G$ and $(F h, h) \geqq 0, h \in \mathfrak{W}$. For let $F$ have the given properties; then

$$
F=\int_{0}^{\infty} \lambda d E_{\lambda}(F)=\int_{0}^{\infty} \mu^{1 / 2} d E_{\mu^{1 / 2}}(F)
$$

and

$$
F^{2}=\int_{0}^{\infty} \mu d E_{\mu^{1 / 2}}(F)=\int_{0}^{\infty} \mu d E_{\mu}(G)
$$

since $F^{2}=G$. Since there is but one resolution of the identity corresponding to a self-adjoint transformation, $E_{\mu 1 / 2}(F)=E_{\mu}(G), 0 \leqq \mu<\infty$. Referring to (12), we have $F$ expressed as that function of $G$ which corresponds to the numerical function $\mu^{1 / 2}$; in other words, $F$ is uniquely determined. This proof is valid in $\mathfrak{E}$ and a fortiori in any manifold in which $F$ is self-adjoint. Thus the relation (11) is valid.

From (10) we deduce

$$
\begin{aligned}
A & =\int_{0}^{1} R(\lambda)\left(\lambda-\lambda^{2}\right)^{1 / 2} d E_{\lambda}(D)=\int_{a_{i-1}}^{a_{i}} R(\lambda)\left(\lambda-\lambda^{2}\right)^{1 / 2} d E_{\lambda}(D) \\
& =\int_{a_{i}-1}^{a_{i}} \frac{\left(\lambda-\lambda^{2}\right)^{1 / 2}}{1-\lambda} d E_{\lambda}(D) \text { in } \mathfrak{N}_{i}(i \geqq 1) .
\end{aligned}
$$

Now let $i \leqq 0$. Keeping in mind that $D=-E+B$ for this case, we see that if $S(\lambda)$ represents the continuous function which equals $(1+\lambda)^{-1}$ for $a_{i-1} \leqq \lambda \leqq a_{i}$ and which is constant otherwise,

$$
B S(D)=S(D) B=E \text { in } \mathfrak{R}_{i}(i \leqq 0) .
$$

Furthermore, since in this manifold $(C h, h) \leqq 0$,

$$
C=\int_{-1}^{0}-\left(-\lambda-\lambda^{2}\right)^{1 / 2} d E_{\lambda}(D) \text { in } \mathfrak{R}_{i}(i \leqq 0) .
$$

In view of (5) we obtain by a reasoning similar to that used above

$$
\begin{aligned}
A & =\int_{-1}^{0}-S(\lambda)\left(-\lambda-\lambda^{2}\right)^{1 / 2} d E_{\lambda}(D)=\int_{a_{i-1}}^{a_{i}}-S(\lambda)\left(-\lambda-\lambda^{2}\right)^{1 / 2} d E_{\lambda}(D) \\
& =\int_{a_{i-1}}^{a_{i}} \frac{-\left(-\lambda-\lambda^{2}\right)^{1 / 2}}{1+\lambda} d E_{\lambda}(D) \text { in } \mathfrak{N}_{i}(i \leqq 0) .
\end{aligned}
$$

Let $T(\lambda)$ be any real function continuous on the open interval $-1<\lambda<1$. We define the symbol 


$$
T(D)=\int_{-1}^{1} T(\lambda) d E_{\lambda}(D)
$$

in such a way as to represent a self-adjoint transformation. The transformation in question is that which in the manifold $\mathfrak{N}_{i}$ introduced above is given by the integral $\int_{a_{i-1}}^{a_{i}} T(\lambda) d E_{\lambda}(D)$. The meaning of the latter has already been made clear since $T(\lambda)$ is continuous over the closure of the range of integration. $T(D)$ is now defined to be the unique transformation whose existence follows from our lemma. Clearly, $T(D)$ does not depend on the choice of the numbers $a_{i}$.

In particular, let $T(\lambda)$ be the monotonic continuous function defined by the equations

$$
\begin{array}{rrr}
T(\lambda)=\frac{-\left(-\lambda-\lambda^{2}\right)^{1 / 2}}{1+\lambda}, & -1<\lambda \leqq 0, \\
T(\lambda)=\frac{\left(\lambda-\lambda^{2}\right)^{1 / 2}}{1-\lambda}, & 0<\lambda<1 .
\end{array}
$$

By (13) and (14), $T(D)=A$ in $\mathfrak{N}_{i},-\infty<i<\infty$. Thus

$$
A=\int_{-1}^{1} T(\lambda) d E_{\lambda}(D) .
$$

Since $T(\lambda)$ is monotonic continuous, it possesses an inverse having the same properties, and we may write in (15)

$$
\mu=T(\lambda), \quad \lambda=V(\mu), \quad \text { and } \quad E_{\lambda}(D)=E_{\mu}(A) .
$$

We thus obtain the customary integral representation of $A$ (1).

We may point out that formula (15) along with the equations of change of variable following it may be used to develop an operational calculus based on $A$. That is, if we assume that the operational calculus based on $D$ has been elaborated, that based on $A$ can be deduced in short order along lines which we need not describe here.

The Francis Joseph University,

SZEGED, Hungary

COLUMBIA UNIVERSITY,

NEW YoRk, N. Y. 